\title{
Evaluation of Clinical Factors Associated with Adverse Drug Events in Patients Receiving Sub-Anesthetic Ketamine Infusions
}

This article was published in the following Dove Press journal:

Journal of Pain Research

\author{
Alexander D Stoker' \\ David M Rosenfeld' \\ Matthew R Buras ${ }^{2}$ \\ Jeremy M Alvord' \\ Andrew W Gorlin' \\ 'Department of Anesthesiology and \\ Perioperative Medicine, Mayo Clinic \\ Arizona, Phoenix, AZ, USA; ${ }^{2}$ Mayo Clinic \\ Arizona, Phoenix, AZ, USA
}

Introduction: Sub-anesthetic ketamine is frequently used as an analgesic to reduce perioperative opioid consumption and has also been shown to have antidepressant effects. Side effects of ketamine include dizziness, diplopia, nystagmus, and psychomimetic effects. It is unclear what clinical factors may be associated with ketamine-related adverse drug events (ADEs).

Methods: We performed a retrospective review of 95 patients who received sub-anesthetic ketamine infusions at our institution. Data examined associations between ketamine-related ADEs and various clinical characteristics including chronic pain, depression, or psychiatric disorder, patient physical characteristics, chronic opioid use, perioperative opioid use, dose and duration of ketamine infusions, pain scores, and perioperative medications such as serotonergic agents, central nervous system (CNS) depressants, and analgesics.

Results: Overall incidence of ketamine-related ADEs was $29.5 \%$ and the incidence of psychomimetic effects was $14.8 \%$. We observed that patients with a history of depression have a lower incidence of ketamine-related ADEs compared to patients without a history of depression $(10.3 \%$ vs $37.3 \%$; $p$ value $=0.007)$.

Conclusion: Patients with depression were found to have a statistically significant reduction in the incidence of ketamine-related ADEs. We found no statistically significant positive associations between ketamine-related ADEs and other clinical factors such as a history of chronic pain, psychiatric disease, patient physical characteristics, perioperative opioid use, dose of ketamine infusion, or co-administration of other CNS depressants.

Keywords: ketamine, pain, depression, adverse, drug-event

\section{Introduction}

Ketamine is an $N$-methyl-D-aspartate (NMDA) receptor antagonist that has been used as an anesthetic for decades. Ketamine is frequently used at sub-anesthetic doses $(<0.5 \mathrm{mg} / \mathrm{kg})$ as an analgesic in perioperative and acute care settings. ${ }^{1,2}$ Ketamine also has anti-depressant effects and there is a substantial body of literature emerging to support its use in the treatment of major depressive disorder (MDD). ${ }^{3}$ In fact, functional MRI data suggests a clear connection between MDD and chronic pain, and ketamine's efficacy for both conditions may point to common mechanistic pathways. Strigo et al (2008) demonstrate that patients with depression cannot modulate pain as effectively as non- depressed patients. ${ }^{4}$ Furthermore, when exposed to standardized painful stimuli, ketamine infusions reduce pain response in areas of the brain such as the anterior cingulate cortex that are well known to modulate the affective components of pain. ${ }^{5}$
Correspondence: Alexander D Stoker Department of Anesthesiology and Perioperative Medicine, Mayo Clinic Arizona, 5777 E Mayo Blvd, Phoenix, AZ 85054, USA

$\mathrm{Tel}+\mid$ 480-510-7933

Email Stoker.Alexander@mayo.edu 
In the perioperative setting, sub-anesthetic ketamine is used most commonly as a single bolus for analgesia either in the operating room or the recovery area or as a continuous infusion (typically $<0.3 \mathrm{mg} / \mathrm{kg} / \mathrm{hr}$ ) that can be administered postoperatively to select patients. ${ }^{6}$ Multiple prospective trials as well as large systematic reviews and meta-analyses support its use for treating pain and reducing opioid consumption in the perioperative period. ${ }^{7,8}$ According to consensus guidelines, intravenous ketamine infusions are indicated for treating patients who are undergoing painful procedures, especially patients with opioid tolerance or dependence. ${ }^{6}$ Due to concerns about side effects, uncertainty exists regarding patient selection for postoperative ketamine infusion.

At sub-anesthetic doses, the most common side effects of ketamine are dizziness, nystagmus, diplopia, and psychomimetic effects including hallucinations, vivid dreams, dysphoria, or a feeling of dissociation. ${ }^{9}$ Of most concern are the psychomimetic effects, and there is uncertainty about the incidence of these events. A large meta-analysis of prospective trials reported an incidence of $7.4 \% ;,{ }^{7,8}$ however, a more recent retrospective analysis reported "central nervous system (CNS) excitation" in $16.2 \%$ of patients. ${ }^{9}$ Sedation is also a concern, with an incidence of $9.4 \%$ noted in a retrospective study by Schwenk et al. (2016). ${ }^{9}$

It is also not known which clinical factors may be associated with the development of ketamine -related adverse drug events (ADEs). Given the discrepancies between the prospective trials and retrospective data, we hypothesize that ketamine-related ADEs may be associated with specific clinical factors such as the presence of chronic pain, psychiatric disorders or co-administration of other CNS depressants which are common in this patient population and excluded in controlled prospective trials. For example, patients in many prospective trials were not treated with gabapentinoids, whereas, in clinical settings, many patients receiving ketamine infusions are also receiving gabapentinoids, and co-administration of these drugs could theoretically increase the risk of ketamine-related ADEs.

This study is a retrospective review of 95 patients who received sub-anesthetic ketamine infusions at our institution over the course of 12 months. The objective is to: (1) examine the clinical characteristics of patients who receive ketamine infusions; (2) evaluate the incidence of ketamine-related ADEs; and (3) determine if there are any associations between ketamine ADEs and specific concomitant diagnoses including chronic pain and psychiatric disorders, as well as the coadministration of other CNS depressants.

\section{Methods}

This study was approved by the Mayo Clinic institutional review board (IRB \# 17-004334) as a minimal risk protocol without requirement for written patient consent and all patient data were maintained with confidentiality.

Inclusion criteria was all patients who received low-dose ketamine infusions for perioperative analgesia or for management of uncontrolled pain in the treatment of a chronic pain syndrome between July 1, 2016 and June 30, 2017 at Mayo Clinic Arizona. Patients were excluded if they had an intrathecal drug infusion pump for chronic pain or if they only received ketamine during procedures in the intensive care unit without a continuous low-dose infusion.

Data examined associations between ketamine-related ADEs and various clinical characteristics including chronic pain, depression, or psychiatric disorder, patient physical characteristics, chronic opioid use, perioperative opioid use, dose and duration of ketamine infusions, pain scores, and perioperative medications such as serotonergic agents, CNS depressants, and analgesics. Any ketamine-related ADE (documented in physician progress notes, nursing notes, or provider notifications) was recorded and specified if it was hallucination, dysphoria, dizziness, visual disturbance, or sedation.

A chi-squared test was used to compare the incidence of ketamine-related ADE among patients who either had a specific preoperative diagnosis (chronic pain, depression, bipolar disorder or psychiatric disorder) or did not. For each variable looked at as a possible contributing factor to ketamine-related ADEs, a Fisher Exact test was used to compare categorical variables and a Kruskal-Wallis test was used to compare the distribution of a numerical variable between groups. A logistic regression model was also performed to test the relationship between ketaminerelated $\mathrm{ADE}$ and depression while controlling for confounding factors, specifically preoperative use of a selective serotonin reuptake inhibitor (SSRI), serotonin and norepinephrine reuptake inhibitor (SNRI), tricyclic antidepressant (TCA), or trazodone. All analyses were completed using RStudio v1.1442 (RStudio, Inc., Boston, MA). All hypothesis tests were two-sided and $p$-values less than 0.05 were considered statistically significant.

\section{Results}

Ninety-five patients met criteria and received a low-dose ketamine infusion during the study interval. Patient demographics of those included in the study are summarized in 
Table 1. Of the 95 patients, 52 were taking chronic opioids at the time of ketamine infusion. The average morphineequivalent daily dose of all patients included in the study was $83.9 \mathrm{mg}$ (Table 2).

\section{Procedures Associated with Low-Dose Ketamine Infusion}

The majority underwent abdominal surgery (55 patients, Table 1). There were six patients who did not undergo a surgical procedure and received low-dose ketamine infusion on hospital admission to treat uncontrolled pain associated with a chronic pain condition.

\section{Medications Taken by Patients Receiving Ketamine Infusions}

Pharmaceutical agents taken by patients while receiving low-dose ketamine infusions are summarized in Table 2. Fifty-two patients received gabapentin or pregabalin, 28 received an SSRI, SNRI, TCA or trazodone, and 22 received a benzodiazepine.

Table I Demographics of Patients Receiving Low-Dose Ketamine Infusions

\begin{tabular}{|l|l|l|}
\hline Prior Diagnosis & $\begin{array}{l}\text { Chronic pain } \\
\text { Depression } \\
\text { Psychotic disorder }\end{array}$ & $\begin{array}{l}46(48.4 \%) \\
29(30.5 \%) \\
2(2.1 \%)\end{array}$ \\
\hline Type of procedure & $\begin{array}{l}\text { No procedure } \\
\text { Abdominal surgery } \\
\text { Spine surgery }\end{array}$ & $\begin{array}{l}6(6.3 \%) \\
55(57.9 \%) \\
25(26.3 \%)\end{array}$ \\
& Orthopedic surgery & $6(6.3 \%)$ \\
& Urology procedure & $1(1.1 \%)$ \\
& Gynecologic surgery & $1(1.1 \%)$ \\
& Head and neck surgery & $1(1.1 \%)$ \\
\hline Average age (years) & & 53.9 \\
\hline Gender & Male & $38(40 \%)$ \\
& Female & $57(60 \%)$ \\
\hline Average weight, BMI & & $79.9 \mathrm{~kg}, \mathrm{BMI}$ \\
& & 27.7 \\
\hline ASA class & $\mathrm{I}$ & $5(5.3 \%)$ \\
& 2 & $45(47.9 \%)$ \\
& 3 & $2(2.1 \%)$ \\
\hline Patients on opioid & 4 & $52(54.7 \%)$ \\
therapy prior to infusion & & \\
\hline
\end{tabular}

Abbreviation: ASA, American Society of Anesthesiologists.
Table 2 Perioperative Medications Administered to Patients While Receiving Low-Dose Ketamine Infusion

\begin{tabular}{|l|l|l|}
\hline $\begin{array}{l}\text { Avg. duration of ketamine } \\
\text { infusion (hrs) }\end{array}$ & 39.4 \\
\hline $\begin{array}{l}\text { Avg. ketamine infusion rate } \\
\text { (mg/hr) }\end{array}$ & & 12.2 \\
\hline $\begin{array}{l}\text { Reason for ketamine } \\
\text { infusion discontinuation }\end{array}$ & $\begin{array}{l}\text { Pain controlled, } \\
\text { transitioned to oral } \\
\text { medication }\end{array}$ & 80 (84.2\%) \\
\cline { 2 - 3 } & Ketamine-related ADE & $14(15 \%)$ \\
\hline $\begin{array}{l}\text { Patients receiving } \\
\text { gabapentin or pregabalin }\end{array}$ & $52(55.3 \%)$ \\
\hline $\begin{array}{l}\text { SSRI, SNRI, TCA, or } \\
\text { trazodone }\end{array}$ & & $28(29.5 \%)$ \\
\hline Benzodiazepines & $22(23.2 \%)$ \\
\hline $\begin{array}{l}\text { Muscle relaxant } \\
\text { (cyclobenzaprine, baclofen, } \\
\text { carisoprodol, } \\
\text { methocarbamol) }\end{array}$ & $22.1 \%)$ \\
\hline Dexmedetomidine & & $13(13.7 \%)$ \\
\hline
\end{tabular}

Abbreviations: ADE, adverse drug event; SSRI, selective serotonin reuptake inhibitor; SNRI, serotonin and norepinephrine reuptake inhibitor; TCA, tricyclic antidepressant.

\section{Characteristics of Ketamine Infusions and Ketamine-Related Adverse Drug Events}

The average ketamine infusion rate was $12.2 \mathrm{mg} / \mathrm{hr}$ with an average duration of $39.4 \mathrm{hrs}$. The reason for ketamine infusion discontinuation was because of adequate pain control and transition to oral pain medication in 80 patients. Infusion was discontinued because of a ketaminerelated ADE in 14 patients. Overall, 28 of the 95 patients experienced a ketamine-related ADE. These ketaminerelated ADEs are summarized in Table 3. A comparison

Table 3 Ketamine-Related ADEs Occurring in Patients Receiving Low-Dose Ketamine Infusions

\begin{tabular}{|l|l|l|}
\hline Incidence of Ketamine-Related ADE & Overall & $28(29.5 \%)$ \\
& Hallucinations & $9(9.5 \%)$ \\
& Dysphoria & $5(5.3 \%)$ \\
& Dizziness & $10(10.5 \%)$ \\
& Visual disturbance & $3(3.2 \%)$ \\
& Sedation & $5(5.3 \%)$ \\
\hline
\end{tabular}

Abbreviation: ADE, adverse drug event. 
of patients who experienced a ketamine-related ADE and patients who did not experience a ketamine-related ADE was performed and is summarized in Table 4.

Eighty-five per cent of infusions were discontinued because pain was controlled and the patient was being transitioned to oral pain medications, and $15 \%$ were discontinued because of ketamine-related ADEs. Of those discontinued because of ketamine-related ADEs, six were stopped during hours $0-24$, four between hours 24 and 48, three between hours 48 and 72, and one between hours 72 and 96. Mean average opioid doses and pain scores during the treatment period are listed in Table 4.

\section{Associations of Ketamine-Related Adverse Drug Events and Depression}

Of the 95 patients included in the study, 29 had a diagnosis of depression at the time of receiving a ketamine infusion. Of the 29 patients with a diagnosis of depression, three experienced a ketamine-related ADE (10.3\%), while 25 of the 66 patients without a diagnosis of depression (37.3\%) experienced a ketamine ADE, which was statistically significant $(p=0.007)$.

A subgroup analysis was performed on patients with and without a diagnosis of depression to assess differences in these two populations (Table 5). Patients with a history of depression were more likely to have received a SSRI,

Table 4 Comparison of Patients Who Experienced A Ketamine-Related ADE and Those Patients Who Did Not Experience A Ketamine-Related ADE

\begin{tabular}{|c|c|c|c|}
\hline & $\begin{array}{l}\text { Patients Who Experienced } \\
\text { Ketamine-Related ADE }(n=28)\end{array}$ & $\begin{array}{l}\text { Patients Who Did Not Experience } \\
\text { Ketamine-Related ADE }(n=67)\end{array}$ & $P$ Value \\
\hline Patients with diagnosis of depression & $10.3 \%(3)$ & $37.3 \%(25)$ & $0.007^{\mathrm{a}}$ \\
\hline Patients with diagnosis of chronic pain & $30.4 \%(14)$ & $47.8 \%(32)$ & $0.101^{\mathrm{a}}$ \\
\hline Patients with diagnosis of psychotic disorder & 1 & 1 & $0.505^{\mathrm{a}}$ \\
\hline Average age (years) & 53 & 54.2 & $0.954^{\mathrm{a}}$ \\
\hline Percent male & $32.1 \%(9)$ & $43.3 \%(29)$ & $0.364^{\mathrm{b}}$ \\
\hline Percent female & $67.9 \%(19)$ & $56.7 \%(38)$ & $0.364^{b}$ \\
\hline Average weight (kg) & 77.2 & 81.0 & $0.453^{\mathrm{a}}$ \\
\hline Average BMI & 26.9 & 28.1 & $0.445^{\mathrm{a}}$ \\
\hline Average ASA class & 2.32 & 2.48 & $0.725^{\mathrm{b}}$ \\
\hline Percent on chronic opioids prior to infusion & $53.6 \%(15)$ & $55.2 \%(37)$ & $1.000^{\mathrm{b}}$ \\
\hline Average daily MEDD (mg) & 109.9 & 73.1 & $0.663^{\mathrm{a}}$ \\
\hline Average ketamine dose $(\mathrm{mg} / \mathrm{hr})$ & 12.1 & 12.3 & $0.686^{\mathrm{a}}$ \\
\hline Average intraop. opioid use (Morphine Eq.) & 57.1 & 66.8 & $0.394^{\mathrm{a}}$ \\
\hline Average opioid use first $24 \mathrm{hr}$ (Morphine Eq.) & 292.4 & 206.1 & $0.193^{\mathrm{a}}$ \\
\hline Average opioid use $24-48$ hrs (Morphine Eq.) & 293.8 & 179.9 & $0.069^{\mathrm{a}}$ \\
\hline Average opioid use $48-72$ hrs (Morphine Eq.) & 283.4 & 160.3 & $0.173^{\mathrm{a}}$ \\
\hline Average postop. total ketamine dose (mg) & 634.0 & 500.0 & $0.877^{\mathrm{a}}$ \\
\hline Average duration of ketamine infusion (hrs) & 44.3 & 37.3 & $0.493^{\mathrm{a}}$ \\
\hline Avg. pain score in PACU or at admission & 7.4 & 7.4 & $0.795^{\mathrm{a}}$ \\
\hline Avg. pain score at 6 hrs & 5.5 & 5.7 & $0.899^{\mathrm{a}}$ \\
\hline Avg. pain score at $12 \mathrm{hrs}$ & 4.9 & 5.0 & $0.987^{\mathrm{a}}$ \\
\hline Avg. pain score at $24 \mathrm{hrs}$ & 4.5 & 5.0 & $0.331^{\mathrm{a}}$ \\
\hline Avg. pain score at $48 \mathrm{hrs}$ & 4.3 & 4.3 & $0.936^{\mathrm{a}}$ \\
\hline Avg pain score at $72 \mathrm{hrs}$ & 4.2 & 3.9 & $0.928^{\mathrm{a}}$ \\
\hline Percent receiving gabapentin or pregabalin & $50 \%(14)$ & $56.7 \%(38)$ & $0.507^{b}$ \\
\hline Percent receiving SSRI, SNRI, TCA, or trazadone & $25 \%(7)$ & $3 \mathrm{I} .3 \%(2 \mathrm{I})$ & $0.627^{\mathrm{b}}$ \\
\hline Percent receiving benzodiazepine & $14.3 \%(4)$ & $26.9 \%(18)$ & $0.286^{\mathrm{b}}$ \\
\hline Percent receiving muscle relaxant & $25 \%(7)$ & $20.9 \%(14)$ & $0.787^{b}$ \\
\hline Dexmedetomidine & $14.3 \%(4)$ & $13.4 \%(9)$ & $1.000^{\mathrm{b}}$ \\
\hline
\end{tabular}

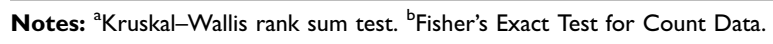

Abbreviations: BMI, body mass index; ADE, adverse drug event; MEDD, morphine-equivalent daily dose; PACU, post-anesthesia care unit; SSRI, selective serotonin reuptake inhibitor; SNRI, serotonin and norepinephrine reuptake inhibitor; TCA, tricyclic antidepressant. 
Table 5 Comparison of Patients with Diagnosis of Depression at the Time of Receiving Low-Dose Ketamine Infusions and Patients with No Diagnosis of Depression

\begin{tabular}{|c|c|c|c|}
\hline & $\begin{array}{l}\text { Patients with } \\
\text { Depression }(n=29)\end{array}$ & $\begin{array}{l}\text { Patients Without } \\
\text { Depression }(n=66)\end{array}$ & $P$ Value \\
\hline Average age (years) & 53.3 & 54.1 & 0.67 \\
\hline Percent male & 31 & 43.9 & 0.364 \\
\hline Percent female & 69 & 56.1 & \\
\hline Average weight (kg) & 79.5 & 80.1 & 0.57 \\
\hline Average BMI & 27.7 & 27.7 & 0.79 \\
\hline Average ASA class & 2.6 & 2.4 & 0.278 \\
\hline Percent on chronic opioids prior to infusion & 55 & 54.5 & 1.0 \\
\hline Average daily MEDD (mg) & 77.9 & 86.6 & 0.92 \\
\hline Average ketamine dose $(\mathrm{mg} / \mathrm{hr})$ & 12.9 & 11.9 & 0.30 \\
\hline Average intraop. opioid use (Morphine Eq.) & 57.5 & 66.8 & 0.23 \\
\hline Average opioid use first $24 \mathrm{hr}$ (Morphine Eq.) & 219.8 & 236.7 & 0.65 \\
\hline Average opioid use $24-48$ hrs (Morphine Eq.) & 183.7 & 226.5 & 0.61 \\
\hline Average opioid use 48-72 hrs (Morphine Eq.) & 154.2 & 215.2 & 0.53 \\
\hline Average postop. total ketamine dose (mg) & 556.7 & 531.8 & 0.57 \\
\hline Average duration of ketamine infusion (hrs) & 39.1 & 39.5 & 0.97 \\
\hline Avg. pain score in PACU or at admission & 8.1 & 7.1 & 0.12 \\
\hline Avg. pain score at $6 \mathrm{hrs}$ & 6.4 & 5.3 & 0.07 \\
\hline Avg. pain score at $12 \mathrm{hrs}$ & 5.7 & 4.7 & 0.08 \\
\hline Avg. pain score at $24 \mathrm{hrs}$ & 5.3 & 4.7 & 0.24 \\
\hline Avg. pain score at $48 \mathrm{hrs}$ & 4.9 & 4.1 & 0.17 \\
\hline Avg. pain score at $72 \mathrm{hrs}$ & 4.3 & 4.0 & 0.59 \\
\hline Percent receiving gabapentin or pregabalin & 62.1 & 51.5 & 0.5 \\
\hline Percent receiving SSRI, SNRI, TCA, or trazadone & 62.1 & 15.2 & $<0.001$ \\
\hline Percent receiving benzodiazepine & 27.6 & 21.2 & 0.60 \\
\hline Percent receiving muscle relaxant & 31.0 & 18.2 & 0.19 \\
\hline dexmedetomidine & 13.8 & 13.6 & 1.0 \\
\hline
\end{tabular}

Abbreviations: ASA, American Society of Anesthesiologists; BMI, body mass index; ADE, adverse drug event; MEDD, morphine-equivalent daily dose; PACU, postanesthesia care unit; SSRI, selective serotonin reuptake inhibitor; SNRI, serotonin and norepinephrine reuptake inhibitor; TCA, tricyclic antidepressant.

SNRI, TCA or trazodone during ketamine infusion $(62.1 \%$ vs $15.2 \%)(p<0.001)$.

Logistic regression analysis was performed on patients with a history of depression. The odds ratio (OR) was 0.189 (95\% CI $0.042-0.610)$ of patients with a history of depression developing a ketamine-related ADE. Adjusted logistic regression analysis was performed to address the possible confounding factor of antidepressants and patients with a history of depression. The OR of ketamine-related ADE in patients with a history of depression was found to be statistically significant at 0.177 (95\% CI 0.139-0.576), while the OR of ketamine-related ADE in patients taking SSRIs, SNRIs, TCAs, or trazodone was not significant at 1.497 (95\% CI 0.484-5.252).

\section{Discussion}

The patients in this study are complex (Table 1), mostly American Society of Anesthesiologists (ASA) class 2 and
3, and they carry a high incidence of conditions such as chronic pain, chronic opioid therapy, and depression that predict challenging postoperative pain management. Coadministration of other CNS depressants commonly used as adjuncts for pain management was also very common.

Most of the above characteristics are comparable with the patient characteristics reported in another recent descriptive analysis of an institution's ketamine infusion practice (Schwenk et al . 2016, RAPM). ${ }^{9}$ That particular study, however, did not report on the co-administration of other CNS depressants. Notably, all of the patients in that particular study had undergone spine surgery, whereas in the present study, the most common surgery type was abdominal surgery (57.9\%) followed by spine surgery $(26.3 \%)$ and orthopedic surgery (6.3\%). In addition, six patients in our study were not surgical patients, but rather chronic pain patients admitted on medicine services for a variety of conditions. 
Ketamine-related ADEs were common with an overall incidence of $29.5 \%$ (Table 3). Combined incidence of psychomimetic effects (hallucinations plus dysphoria) was $14.8 \%$. In Table 6 we compare the incidence of ketamine-related ADEs in our study with those reported by Schwenk et al. (2016) as well as those reported in two large meta-analyses of prospective trials of perioperative ketamine. Overall, the incidence of ketamine-related ADEs reported in our study as well as that by Schwenk et al. (2016) is significantly higher than that reported in the two meta-analyses. In addition, sedation in particular was reported in $5.3 \%$ of the patients in this study and in $9.4 \%$ by Schwenk et al. (2016), whereas sedation is infrequently reported as a side effect in most of the prospective trials. In fact, Elia et al. (2005) suggest that ketamine has a protective effect against sedation, presumably via its opioid-sparing effect. What can explain these discrepancies?

First of all, it is important that all of the patients in our study as well as the study by Schwenk et al. (2016) received postoperative ketamine infusions in addition to intraoperative ketamine. The prospective trials analyzed by Elia et al. (2005) and Laskowski et al. (2011) were heterogeneous, some involved postoperative ketamine infusions; however, many of the studies had protocols that involved only intraoperative ketamine. It is reasonable to suggest that postoperative infusions could increase the risk of ketamine-related ADEs (and sedation in particular) as compared with intraoperative therapy alone. Although this proposition has not been studied in a controlled prospective trial, our data do offer some limited evidence to support that hypothesis.

Table 6 Comparison of the Incidence of Ketamine-Related ADEs Seen in Our Study with Other Retrospective and Meta-Analysis of Prospective Studies

\begin{tabular}{|l|l|l|l|l|}
\hline & $\begin{array}{l}\text { Stoker } \\
\text { et al. }\end{array}$ & $\begin{array}{l}\text { Schenk } \\
\text { et al. }\end{array}$ & $\begin{array}{l}\text { Laskowski } \\
\text { et al. }\end{array}$ & $\begin{array}{l}\text { Elia } \\
\text { et al. }\end{array}$ \\
\hline Overall & $29.50 \%$ & $31.80 \%$ & N/A & N/A \\
Psychomimetic effects & $14.80 \%$ & $16.20 \%$ & $7.35 \% *$ & $7.40 \%$ \\
Ocular/visual effects & $3.20 \%$ & $3.10 \%$ & $*$ & $6.20 \%$ \\
Dizziness & $10.50 \%$ & N/A & N/A & $* * *$ \\
Sedation & $5.30 \%$ & $9.40 \%$ & $2.53 \% * *$ & \\
Nightmares & & & & $2.40 \%$ \\
\hline
\end{tabular}

Notes: *Ocular and visual effects included with psychomimetic effects. **imilar incidence as seen in patient who did not receive ketamine. *** Incidence of sedation not reported, although meta-analysis reported a relative risk of 0.5 of sedation with use of ketamine versus control in patients receiving opioids.
Second, the patients evaluated in prospective trials are not the same patients who received ketamine infusions in our practice. As our demographic data show, patients who receive ketamine in our practice are complex, with a high incidence of psychiatric comorbidities, chronic pain, opioid tolerance, and often take other CNS depressants. In other words, compared with the patients who are entered into most prospective controlled trials, we hypothesize that perhaps these patients are vulnerable to neuropsychiatric side effects.

Despite the logical appeal of this hypothesis, our data do not reveal any statistically significant positive association between any clinical factor with the development of ketaminerelated ADEs (see Table 4). There was a non-statistically significant trend suggesting that higher postoperative opioid dose could be associated with ketamine-related ADEs. In addition, patients who experience ketamine-related ADE had a longer average duration of ketamine infusion and higher cumulative ketamine dose, but these were not statistically significant differences and the average infusion dose in milligrams per hour was similar.

The most interesting finding in our study was a negative correlation between ketamine-related ADEs and a prior diagnosis of depression (10.3\% ADE with depression vs $37.3 \%$ ADE without depression, $p=0.007$ ). Patients with depression were compared with those without depression (see Table 5) and no significant differences were found in other clinical factors other than an unsurprising positive association with depressed patients taking antidepressant medications. We considered the possibility that the antidepressants could be a confounder and possibly providing the protective effect, but we found no independent association (either positive or negative) between antidepressant use and ketamine-related ADEs. The diagnosis of depression appears independently associated with a lower incidence of ketamine-related ADEs.

This finding is intriguing in light of the fact that ketamine itself is a potent antidepressant and an intranasal formulation was recently approved in the United States for treatment of MDD. ${ }^{10}$ Psychomimetic, cardiovascular, and neurological side effects are frequently reported in studies examining the use of ketamine for the treatment of depression. ${ }^{11}$ That said, it is difficult to compare the psychiatric patient population with the perioperative patient population. The dosing protocols in the depression studies are different (often more aggressive) and the patients are being treated for acute major depressive episodes, whereas most of the patients in the perioperative 
environment with a diagnosis of depression have stable chronic disease.

Nonetheless, these findings beg the question, is there something about the depressed patient's neurochemistry or neurocircuitry that exerts a protective effect against ketaminerelated ADEs? The primary in-vivo agonist of the NMDA receptor is glutamate, an excitatory neurotransmitter that interacts with multiple receptors and pathways throughout the CNS. Glutamate and the NMDA receptor are thought to be primary actors in numerous mental and psychiatric disorders, including MDD and schizophrenia. ${ }^{12,13}$ Glutamate levels are higher in the plasma and the cerebral cortex in depressed patients when compared with non-depressed patients. ${ }^{12-16}$ Studies using magnetic resonance spectroscopy to measure brain glutamate in depressed patients demonstrate significant differences in multiple brain regions when compared with non-depressed controls. ${ }^{13,17,18}$ Taken together with the fact that ketamine (which blocks glutamate at the NMDA receptor (NMDAr)) is a potent antidepressant, this suggests that elevated glutamate/NMDAr activity in certain CNS pathways may be involved in MDD.

Kim et al. (1980) demonstrated that cerebrospinal fluid glutamate levels are low in patients with schizophrenia and this led to the glutamate hypofunction hypothesis of schizophrenia. $^{12,14,19}$ This is consistent with the observation that ketamine and phencyclidine, NMDA receptor antagonists, cause dose-dependent psychotic symptoms. Clearly, glutamate and NMDAr are involved in both MDD and psychotic symptoms, although the exact pathways and mechanisms and how they relate to one another are not fully understood. If glutamate/NMDAr activity exists on a continuum, with MDD on the high end and psychosis on the low end, this could explain our finding that depression is protective against ketamine-induced psychomimetic effects (see Figure 1). Finally, MDD and psychosis are complex phenomena that likely involve other neurotransmitter/receptor systems and, likewise, ketamine is known to interact with multiple other receptor types in the CNS (voltage-gated $\mathrm{Na}$

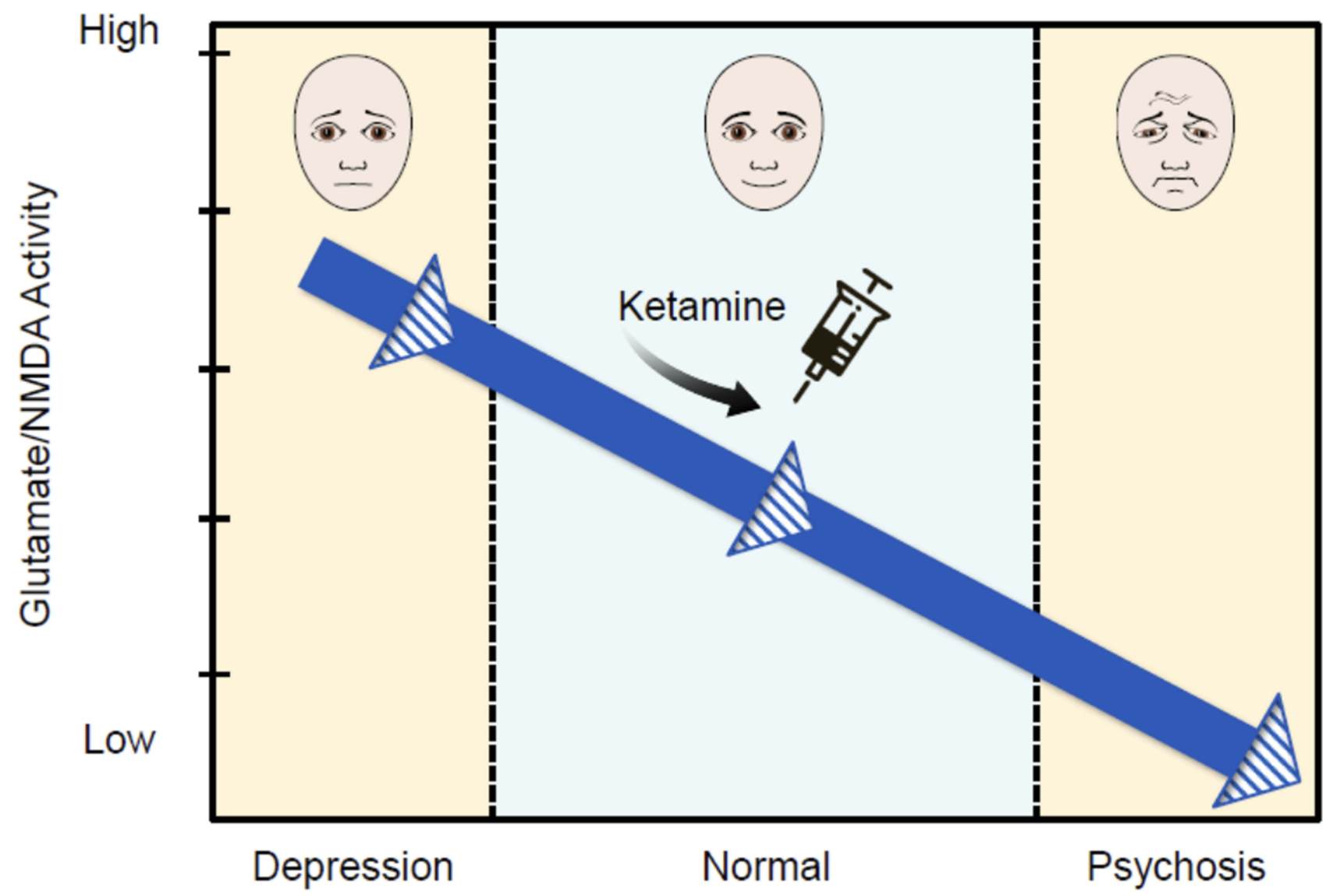

Figure I Hypothetical model explaining relationship of glutamate/NMDA activity in relevant CNS pathways with psychiatric symptoms. In this model, high glutamate/NMDA activity is associated with depression and low glutamate/NMDA activity is associated with psychosis. Ketamine reduces glutamate/NMDA activity in a dose-dependent manner. Depressed patients may have higher baseline glutamate/NMDA activity such that when given ketamine, they do not drop below the theoretical threshold necessary for the development of psychotic symptoms. 
and $\mathrm{K}$ channels, serotonin receptors, inhibition of dopamine reuptake, and others). ${ }^{20}$ It is entirely plausible that other receptors and neural pathways are at least partially responsible for ketamine's interplay with depression and psychomimetic effects.

This finding may potentially have value for surgical patients receiving ketamine infusions. Investigators have looked at ketamine's antidepressant effects in the perioperative setting and the data are conflicting. In a large randomized clinical trial of 670 patients, Mashour et al. (2018) reported that ketamine had no effect on the incidence of postoperative depression in patients without a pre-existing diagnosis of depression. ${ }^{21}$ Perhaps the key patients to look at are patients who do have depression. Kudoh et al. (2002) report that low-dose ketamine does improve postoperative depression in previously depressed patients, although it is a small study and has not been since replicated (A\&A 2002). ${ }^{22}$ Thus, we know that ketamine improves mood in non-surgical and surgical patients and, if our finding of depression's protective effect against ketamine-related ADEs is confirmed, it would suggest that depressed patients are ideal candidates for ketamine therapy in the perioperative setting.

\section{Limitations}

This study is based on retrospective data and a relatively small number of patients. Our patient population may not be representative of other practices, in particular a high proportion of patients undergoing abdominal procedures. In addition, ketamine-related ADEs were analyzed together because the study was not powered to analyze associations with individual ketamine-related ADEs. Finally, there is no control group of patients that did not receive ketamine. Because most of the ADEs included are also possible with opioids, it is possible that the rates of ketamine-related ADEs are overestimated in the present study.

\section{Conclusions}

Patients treated with perioperative ketamine in this study were complex, mostly ASA 2 and ASA 3, with a high incidence of chronic pain, depression, and chronic opioid therapy. The incidence of ketamine-related ADEs was $29.5 \%$ overall and the incidence of psychomimetic effects was $14.8 \%$. These numbers are consistent with another similar retrospective study but significantly higher than that reported in meta-analyses and prospective trials. We found no statistically significant positive associations between ketamine-related ADEs and other clinical factors such chronic pain, chronic opioid therapy, psychiatric disease or co-administration of other CNS depressants. Patients with depression were found to have a statistically significant reduction in the incidence of ketamine-related ADEs. This finding suggests that the depressed patient is less susceptible to ketamine-induced ADEs, hypothetically because of a higher baseline level of glutamate/NMDAr activity in certain areas or pathways within the CNS. Clearly, more research is needed to further delineate this phenomenon.

\section{Author Contributions}

All authors contributed to data analysis, drafting or revising the article, gave final approval of the version to be published, and agree to be accountable for all aspects of the work.

\section{Disclosure}

The authors report no conflicts of interest in this work.

\section{References}

1. Menigaux C, Guignard B, Fletcher D, Sessler DI, Levron JC, Chauvin M. Intraoperative small-dose ketamine enhances analgesia after outpatient knee arthroscopy. Anesth Analg. 2001;93:606. doi:10.1097/00000539-200109000-00016

2. Kwok RF, Lim J, Chan MT, Gin T, Chiu WK. Preoperative ketamine improves postoperative analgesia after gynaecologic laparoscopic surgery. Anesth Analg. 2004;98:1044-1049. doi:10.1213/01.ANE. 0000105911.66089 .59

3. Zarate CA. Ketamine for depression: evidence, challenges and promise. World Psychiatry. 2015;14(3):348-350. doi:10.1002/wps.20269

4. Strigo I, Simmons A, Matthews S, Craig A, Paulus M. Association of major depressive disorder with altered functional brain response during anticipation and processing. Arch Gen Psychiatry. 2008;65 (11):1275-1284. doi:10.1001/archpsyc.65.11.1275

5. Sprenger T, Valet M, Woltmann R, et al. Imaging pain modulation by subanesthetic S-(+)-Ketamine. Anesth Analg. 2006;103:729-737. doi:10.1213/01.ane.0000231635.14872.40

6. Gorlin AW, Rosenfeld DM, Ramakrishna H. Intravenous sub-anesthetic ketamine for perioperative analgesia. J Anaesthesiol Clin Pharmacol. 2016;32:160-167. doi:10.4103/0970-9185.182085

7. Laskowski K, Stirling A, McKay WP, Lim HJ. A systematic review of intravenous ketamine for postoperative analgesia. Can J Anaesth. 2011;58:911-923. doi:10.1007/s12630-011-9560-0

8. Elia N, Tramèr MR. Ketamine and postoperative pain - A quantitative systematic review of randomised trials. Pain. 2005;113:61-70. doi:10.1016/j.pain.2004.09.036

9. Schwenk ES, Goldberg SF, Patel RD, et al. Adverse drug effects and preoperative medication factors related to perioperative low-dose ketamine infusions. Reg Anesth Pain Med. 2016;41:482-487. doi:10.1097/AAP.0000000000000416

10. Kim J, Farchione T, Potter A, Chen Q, Temple R. Esketamine for treatment-resistant depression-first FDA-approved antidepressant in a new class. $N$ Engl J Med. 2019;381:1-4. doi:10.1056/NEJMp1903305

11. Short B, Fong J, Galvez V, Shelker W, Loo C. Side-effects associated with ketamine use in depression a systematic review. Lancet Psychiatry. 2018;5(1):65-78. 
12. Lakhan S, Caro M, Hadzimichalis N. NMDA receptor activity in neuropsychiatric disorders. Font Psychiatry. 2013;4:52.

13. Sanacora G, Zarate CA, Krystal JH, Manji HK. Targeting the glutamatergic system to develop novel, improved therapeutics for mood disorders. Nat Rev Drug Discov. 2008;7:426-437. doi:10.1038/nrd2462

14. Kim J, Kornhuber H, Schmid-Birgk W, Holzmuller B. Low cerebrospinal fluid glutamate in schizophrenic patients and a new hypothesis on schizophrenia. Neurosci Lett. 1980;20(3):379-382. doi:10.1016/03043940(80)90178-0

15. Altamura C, Maes M, Dai J, Meltzer H. Plasma concentrations of excitatory amino acids, serene, glycine, taurine and histidine in major depression. Eur Neuropsychopharmacol. 1995;71-75. doi:10.1016/ 0924-977X(95)00033-L

16. Hashimoto K, Bruno D, Nierenberg $J$, et al. Abnormality in glutamine-glutamate cycle in the cerebrospinal fluid of cognitively intact elderly individuals with major depressive disorder: a 3-year follow-up study. Transl Psychiatry. 2016;6:744. doi:10.1038/tp.2016.8

17. De Graaf R, Mason G, Patel A, Behar K, Rothman D. In vivo 1H-[13C]NMR spectroscopy of cerebral metabolism. NMR Biomed. 2003;16(67):339-357. doi:10.1002/nbm.847
18. Levine $\mathrm{J}$, et al. Increased cerebrospinal fluid glutamine levels in depressed patients. Biol Psychiatry. 2000;47:586-593. doi:10.1016/ S0006-3223(99)00284-X

19. Gilmour G, Dix S, Fellini L, et al. NMDA receptors, cognition and schizophrenia-testing the validity of the NMDA receptor hypofunction hypothesis. Neuropharmacology. 2012;6(3):1401-1412. doi:10.1016/j. neuropharm.2011.03.015

20. Eldufani J, Nekoui A, Blaise G. Nonanesthetic effects of ketamine: a review article. Am J Med. 2018;131(12):1418-1424.

21. Mashour GA, Abdallah AB, Pryor KO, et al. Intraoperative ketamine for prevention of depressive symptoms after major surgery in older adults: an international, multicenter, double-blind, randomized clinical trial. $B r J$ Anesth. 2018;121(5):1075e1083. doi:10.1016/j. bja.2018.03.030

22. Kudoh A, Takahira Y, Katagai H, Takazawa T. Small-dose ketamine improves the postoperative state of depressed patients. Anesth Analg. 2002;95(1):114-118. doi:10.1097/00000539-200207000-00020

23. Kim JS, Schmid-Burgk W, Claus D, Kornhuber HH. Increased serum glutamate in depressed patients. Arch Psychiatr Nervenkr. 1982;232: 299-304. doi:10.1007/BF00345492

\section{Publish your work in this journal}

The Journal of Pain Research is an international, peer reviewed, open access, online journal that welcomes laboratory and clinical findings in the fields of pain research and the prevention and management of pain. Original research, reviews, symposium reports, hypothesis formation and commentaries are all considered for publication. The manuscript management system is completely online and includes a very quick and fair peer-review system, which is all easy to use. Visit http:// www.dovepress.com/testimonials.php to read real quotes from published authors. 